\title{
Kasuistiken
}

Ophthalmologe $2021 \cdot 118: 940-943$ https://doi.org/10.1007/s00347-020-01207-1 Online publiziert: 21. August 2020

(c) Der/die Autor(en) 2020
C. J. Farah (D) - B. Seitz · L. Hamon · C. Sourlis · L. Daas

Klinik für Augenheilkunde, Universitätsklinikum des Saarlandes UKS, Homburg/Saar, Deutschland

\section{Mischinfektionen bei kontaktlinsenassoziierter mykotischer Keratitis mit Pseudomonas oder Akanthamöben}

Pilzkeratitiden sind ein relativ seltenes, aber sehr ernst zu nehmendes Krankheitsbild. Die korrekte Diagnose wird in Deutschland initial nur bei $1 / 5$ der Fälle gestellt $[1,10]$. Die Gefahr für das Auge ist groß, denn nicht adäquat behandelt, kann es zu Hornhautvernarbungen, Augenperforationen oder Endophthalmitiden kommen, was die visuelle Prognose erheblich reduziert und den Erhalt des Auges unsicher macht. Eine perforierende Keratoplastik ist im Verlauf nicht ungewöhnlich. In dem Deutschen Pilz-Keratitis-Register erhielten $65,7 \%$ der eingeschlossenen Patienten im Verlauf der Krankheit eine perforierende Keratoplastik [10].

Die klinische Diagnosestellung einer mykotischen Keratitis stellt uns besonders bei atypischem Krankheitsbild vor Herausforderungen. Eine Kontaktlinsenanamnese lässt uns manchmal a priori zu Unrecht eine solitäre Akanthamöbenkeratitis vermuten. Aufgrund der möglichen Koinfektion sind wir der Meinung, dass alle diagnostischen Modalitäten - Polymerasekettenreaktion (PCR), In-vitro-Kultivierung, Histologie und in vivo konfokale Mikroskopie parallel durchgeführt werden sollten [4, 11]. Bei der therapierefraktären kontaktlinsenassoziierten mykotischen Keratitis muss man nicht selten auch auf Koinfektionen oder Superinfektionen gefasst sein $[8,9]$. Bei Koinfektionen ist es meistens problematisch, rechtzeitig die adäquate Therapie einzuleiten.

\section{Klinischer Befund}

Patientin 1. Eine 37-jährige Kontaktlinsenträgerin stellte sich zur Mitbeurteilung mit seit 5 Tagen zunehmender Visusminderung und Schmerzen bei therapierefraktärer Keratitis in unserer Hochschulambulanz vor. Die Patientin hatte keine Haustiere, und anamnestisch war Kontakt mit kontaminiertem Wasser nicht bekannt. Eine Kombinationstherapie von Tobramycin und DexamethasonAugentropfen sowie lokale und systemische Aciclovir-Gabe (5-mal $400 \mathrm{mg}$ pro Tag) war extern eingeleitet worden. Spaltlampenbiomikroskopisch zeigten sich am linken Auge ein großes stromales Infiltrat mit Epitheldefekt und die Vorderkammer mit einem für mykotische Keratitiden typischen pyramiden-

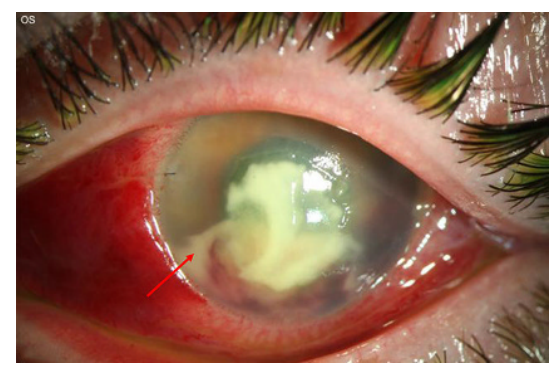

Abb. $1 \Delta$ Patientin 1:2. Tag des stationären Aufenthaltes. Zustand nach Vorderkammerspülung und Hornhautabrasio: zentraler Epitheldefekt mit stromalem Infiltrat, Vorderkammer mit gemischtem Blut und pyramidenförmigem Hypopyon-Rezidiv (BB-1-Zeichen nach Behrens-Baumann, Pfeil) $[2,11]$ förmigen Hypopyon ( $\bullet$ Abb. 1, Bild nach Vorderkammerspülung).

Patientin 2. Eine 25-jährige Kontaktlinsenträgerin stellte sich zur Mitbeurteilung mit seit 4 Wochen bestehender Visusminderung, Lichtempfindlichkeit und Schmerzen bei therapierefraktärer Keratitis in unserer Hochschulambulanz vor. Die Patientin geht in ihrer Freizeit oft reiten, und anamnestisch war kein Kontakt mit kontaminiertem Wasser bekannt. Eine Kombinationstherapie von Moxifloxacin, Polymyxin B, Neomycin und Gramicidin, Dexamethason und antimykotischen (Voriconazol) Augentropfen war extern eingeleitet worden. Spaltlampenbiomikroskopisch zeigte sich am linken Auge eine diffuse interstitielle Keratitis mit ringförmigen multifokal akzentuierten Infiltraten, retrokornealen Beschlägen und einem 2-mm-Hypopyon (• Abb. 2).

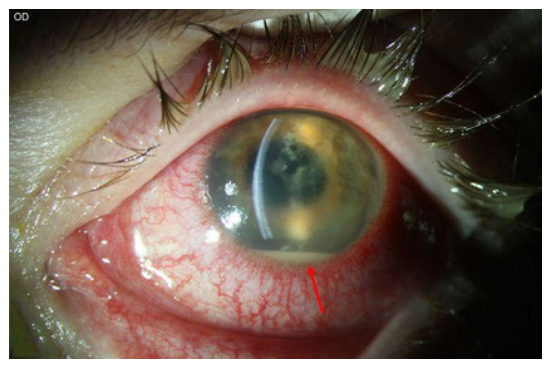

Abb. 2 \ Patientin 2. Aufnahmetag. Spaltlampenbefund mit diffuser interstitieller Keratitis, ringförmigen multifokal akzentuierten Infiltraten und $2 \mathrm{~mm}$ hohem Hypopyon (Pfeil) 

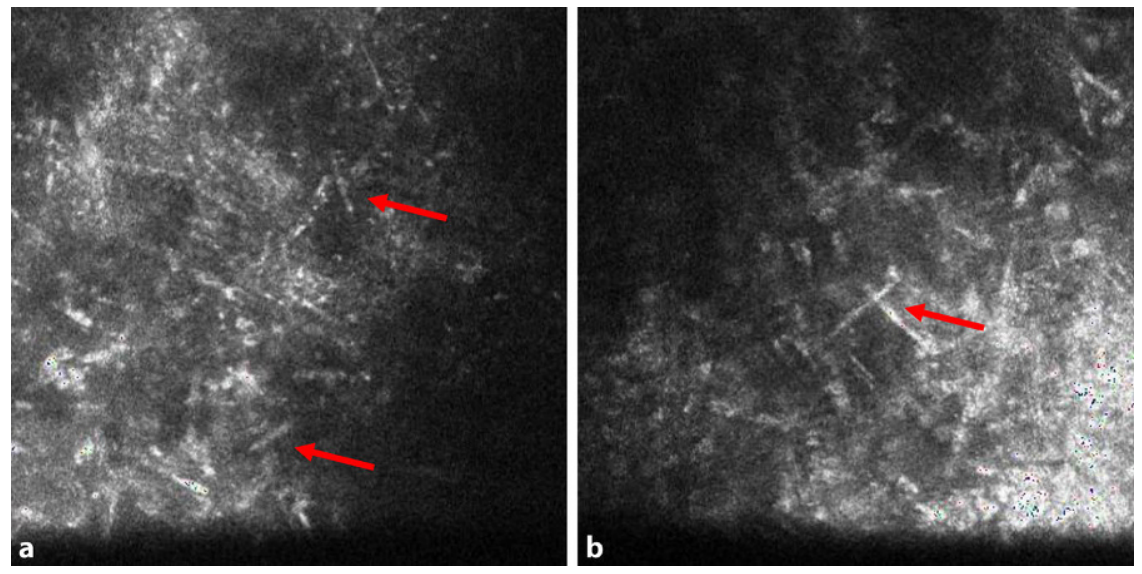

Abb. 3 A Patientin 1. Konfokal mikroskopischer Befund: helle, unterschiedlich breite hyperreflektive Linien im Sinne von Pseudohyphen bei einer mykotischen Keratitis (Pfeile, a, b) [4]
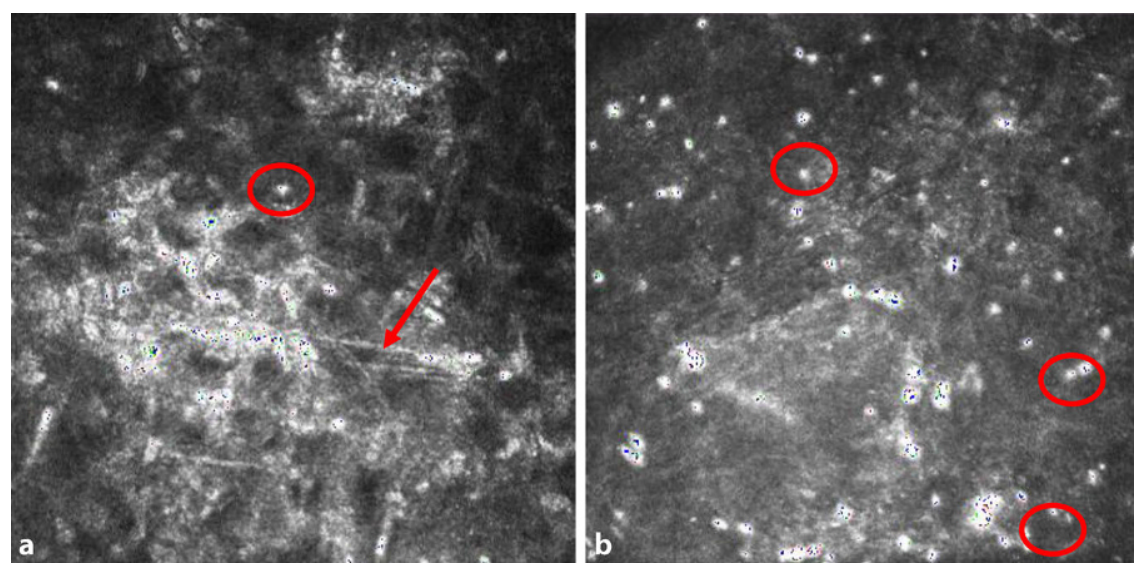

Abb. 4 \ Patientin 2. In vivo konfokale Mikroskopie im Bereich des stromalen Infiltrats: helle, unterschiedlich breite und teilweise parallel verlaufende hyperreflektive Linien im Sinne von Pilzhyphen (Pfeil, a) und Akanthamöben typische rundliche hyperreflektive Strukturen (Kreise, a, b) [4]

\section{Diagnose}

Bei Patientin 1 betrug bei der Aufnahme der bestkorrigierte Visus (BCVA) Handbewegung am betroffenen linken Auge und 0,8 am gesunden rechten Auge. In der Sonographie bestand kein Anhalt für eine Endophthalmitis. Es zeigten sich in der konfokalen Mikroskopie hyperreflektive Strukturen ( $\bullet$ Abb. 3a, b) die für eine mykotische Keratitis sprechen. Eine Hornhautabrasion, Vorderkammerspülung mit Medikamenteingabe (Amphotericin B) und diagnostische Vorderkammeraspiration wurden am gleichen Tag durchgeführt. Bei therapierefraktärem Hypopyon und starkem Verdacht auf Pilzkeratitis führten wir am 3. Tag der Aufnahme eine Keratoplastik à chaud durch. Die PCR war 4 Tage nach der stationären Aufnahme negativ im Vorderkammeraspirat, aber positiv für Pilze im Hornhautabradat. Auch Pseudomonas aeruginosa konnte zeitnah in der In-vitro-Kultivierung nachgewiesen werden. Die PCR der Kontaktlinse und Kontaktlinsenbehälter sicherten am nächsten Tag eine Mischinfektion von Pseudomonas aeruginosa und Candida parapsilosis.

Bei Patientin 2 betrug am Tag der stationären Aufnahme nach einer 4-wöchigen Vorbehandlung der bestkorrigierte Visus (BCVA) 0,1 am betroffenen linken Auge und 1,0 am gesunden rechten Auge. Bis zum Tag der Aufnahme konnten extern keine Erreger nachgewiesen werden. In der Sonographie bestand kein Anhalt für eine Endophthalmitis. In der konfokalen Mikroskopie konnten sowohl hyperreflektive Linien wie bei mykotischer Keratitis als auch Akanth-

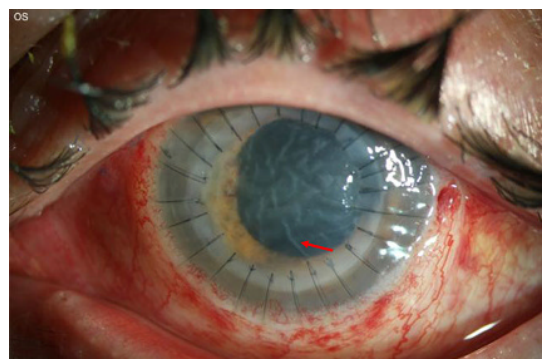

Abb. $5 \Delta$ Patientin 1:6. postoperativer Tag nach perf. Excimerlaser-Keratoplastik à chaud $(8,0 / 8,1 \mathrm{~mm})$, Einzelknüpfnähte fest, zentrale Descemet-Falten (Pfeil)

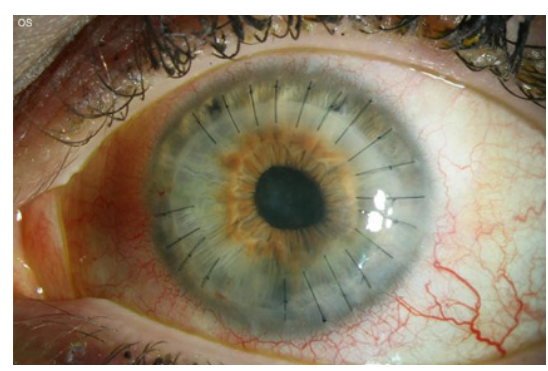

Abb. 6 \ Patientin 2: 3 Monate nach perf. Excimerlaser-Keratoplastik à chaud $(8,5 / 8,6 \mathrm{~mm})$. Die lockeren Hornhautfäden wurden entfernt

amöben-Zysten nachgewiesen werden (- Abb. 4a, b). Eine Hornhautabrasion, Vorderkammerspülung mit Medikamenteingabe (Amphotericin B) und diagnostische Vorderkammeraspiration konnten am nächsten Tag durchgeführt werden. Die PCR sicherte eine Mischinfektion von Pilz (Pilz-DNA nicht weiter differenzierbar) und Akanthamöben im Hornhautabradat. Das Vorderkammeraspirat war negativ, und die In-vitro-Kultivierung konnte 4 Wochen nach einer durchgeführten Keratoplastik à chaud kein Wachstum nachweisen.

\section{Therapie und Verlauf}

Beide Patientinnen erhielten nach intensiver lokaler und systemischer antibakterieller und antimykotischer Therapie eine Excimerlaser-Keratoplastik à chaud (Transplantatdurchmesser bei Patientin 1: $8,0 / 8,1 \mathrm{~mm}$, bei Patientin 2: $8,5 / 8,6 \mathrm{~mm}$; mit 24 Einzelknüpfnähten in beiden Augen) mit mehrmaliger Vorderkammerspülung, Medikamenteingabe (Amphotericin B) und ggf. antiamöboider Therapie im Verlauf. 
Patientin 1 Die lokale Therapie bestand in unserer Klinik aus Voriconazol 2\%, Amphotericin B 1\% und Fortified-Augentropfen (Tobramycin und Cefazolin $5 \%$ ), da beide Keime (Candida und Pseudomonas) dagegen sensibel waren. Die systemische Therapie bestand aus Voriconazol p.o. $200 \mathrm{mg} 1-0-1$, Vancomycin i.v. 1 g 1-0-1, Ceftazidim i.v. 2 g 1-1-1. Die Patientin wurde bei persistierendem Epitheldefekt mit einer Amnionmembrantransplantation als Patch entlassen. Die lokalen antimykotischen und Fortified-Augentropfen wurden über mehr als 2 Monate fortgesetzt mit langsam ausschleichender lokaler und milder systemischer Kortikosteroidtherapie (Therapiestart mit $100 \mathrm{mg}$ oral mit Abstufung $20 \mathrm{mg}$ alle 2 Tage) zur Nachbehandlung der Keratoplastik (• Abb. 5).

Patientin 2 Die lokale Therapie bestand in unserer Klinik aus Voriconazol 2\%, Natamycin 5\%, Amphotericin B 1\%, Polyhexanid, Propamidine isethinate und einem Kombinationspräparat aus Neomycin-, Polymyxin B- und Bacitracin/Gramicidin-Augentropfen. Die systemische Therapie bestand aus Voriconazol 200 mg 1-0-1 und milden Kortikosteroiden (100 mg mit Abstufung $20 \mathrm{mg}$ alle 2 Tage) als Nachbehandlung der Keratoplastik (• Abb. 6). Die lokale Triple-Therapie (Polyhexanid, Propamidine isethinate, Amphotericin B 1\%) wurde über 2 Monate fortgesetzt.

\section{Diskussion}

Diese Doppelkasuistik betont die breite Differenzialdiagnose und die daraus potenziell resultierenden therapeutischen Schwierigkeiten bei kontaktlinsenassoziierten Keratitiden. Fehldiagnosen sind aufgrund atypischer Erscheinungsbilder in der frühen Phase häufig. Jedoch gibt es einige klinische Anzeichen, die den Augenarzt warnen sollten. Eine gründliche Anamnese des Kontakts mit Tieren oder kontaminiertem Wasser steigert die Angst vor Pilz- oder Akanthamöbeninfektionen. Eine Kontaktlinsenanamnese lässt uns manchmal a priori zu Unrecht eine solitäre Akanthamöbenkeratitis vermuten. Eine sehr schnell fortschreitende eitrige stromale Keratitis deutet

Ophthalmologe 2021 · 118:940-943 https://doi.org/10.1007/s00347-020-01207-1

(c) Der/die Autor(en) 2020

\section{J. Farah · B. Seitz · L. Hamon · C. Sourlis · L. Daas}

\section{Mischinfektionen bei kontaktlinsenassoziierter mykotischer Keratitis mit Pseudomonas oder Akanthamöben}

\section{Zusammenfassung}

Kontaktlinsenassoziierte Keratitiden werden immer häufiger. Die mykotische Keratitis ist ein relativ seltenes, aber sehr ernst zu nehmendes Krankheitsbild. Meist wird im Frühstadium eine falsche Diagnose gestellt und dadurch die adäquate Therapie verzögert. Bei der therapierefraktären kontaktlinsenassoziierten mykotischen Keratitis können nicht selten auch Koinfektionen oder Superinfektionen bestehen. Wir stellen 2 Patienten mit initial unklarer Keratitis vor, bei denen eine Mischinfektion der mykoti- schen Keratitis mit Pseudomonas aeruginosa bzw. Akanthamöben nachgewiesen werden konnte. In beiden Fällen war die zeitnahe perforierende Excimerlaser-Keratoplastik mit Einzelknüpfnähten und adäquater Lokaltherapie über 8 Wochen therapeutisch erfolgreich.

Schlüsselwörter

Hornhaut $\cdot$ Kontaktlinse $\cdot$ Pilz $\cdot$ Keratitis . Konfokale Mikroskopie

\section{Coinfections in contact lens-associated mycotic keratitis with Pseudomonas or Acanthamoeba}

\section{Abstract}

Contact lens-associated keratitis is becoming increasingly more frequent. Fungal keratitis is a relatively rare clinical picture but must be taken very seriously. Especially in the early stages of the disease, it may be clinically misdiagnosed and adequate treatment is therefore delayed. In treatment-resistant contact lens-associated fungal keratitis, coinfections or superinfections can occur. We present two patients with an initially unclear keratitis, in whom a fungal keratitis with coinfection of Pseudomonas aeruginosa and Acanthamoeba, respectively, could be confirmed. In both cases an urgent excimer laser penetrating keratoplasty with interrupted sutures and adequate local topical treatment for 8 weeks was successful.

Keywords

Cornea - Contact lens - Fungal keratitis . Confocal microscopy auf eine Pseudomonas-Infektion hin [3]. Pilzkeratitiden können als haloförmiges Infiltrat mit möglichen Satellitenläsionen erscheinen [1, 4, 10]. $\mathrm{Zu}$ diesem Krankheitsbild sind das pyramidenförmige Hypopyon und das Hornhautinfiltrat mit intaktem Epithel assoziiert, in Deutschland auch als BB-1- und BB2-Zeichen nach Behrens-Baumann bekannt [2, 11]. Aus unserer Erfahrung ist der Vorderkammerreizzustand ein kritisches prognostisches Kriterium bei allen mykotischen Keratitiden.

Die wiederkehrenden Akanthamöbeninfektionen werden als Frühzeichen durch das sog. „Dirty-Epithelium“ gekennzeichnet, Schmerzen sind typischerweise stark, aber uneinheitlich [5].

Eine angemessene Behandlung kann nur durch ein systematisches Vorgehen erreicht werden. Aufgrund der möglichen Mischinfektion sind wir der Meinung, dass alle diagnostischen Modalitäten - Polymerasekettenreaktion (PCR), In-vitro-Kultivierung, Histologie und in vivo konfokale Mikroskopie am Aufnahmetag - parallel durchgeführt werden sollten $[4,8,11]$. Konfokale Mikroskopie ist nicht als ein alleinstehendes diagnostisches Verfahren anzusehen, sondern als Ergänzung zu dem Spektrum der diagnostischen Modalitäten besonders bei atypischen Hornhautbefunden. Die Interpretation der konfokalen Mikroskopie hängt von der Expertise des Untersuchers ab [6].

Wertschätzendes interdisziplinäres Zusammenarbeiten mit der Mikrobiologie ist unerlässlich zur Identifizierung der Pathogene und Anpassung der The- 
rapie. Bei Verdacht auf Pilzinfektion (mit unbekanntem Erreger) sollte topisches Voriconazol 2\% als Primärbehandlung eingeleitet werden, denn der antimykotische Wirkstoff kann auch gegen andere Erreger wie Akanthamöben wirken $[1,7]$. In beiden Fällen war die zeitnahe perforierende Excimerlaser-Keratoplastik mit Einzelknüpfnähten und adäquater Lokaltherapie über 8 Wochen therapeutisch erfolgreich.

\section{Fazit für die Praxis}

Bei jeder unklaren oder therapieresistenten kontaktlinsenassoziierten Keratitis sollte an eine Mischinfektion gedacht werden, und eine Polymerasekettenreaktion (PCR), In-vitro-Kultivierung, Histologie und in vivo konfokale Mikroskopie sollten am Aufnahmetag parallel durchgeführt werden.

\section{Korrespondenzadresse}

C. J. Farah
Klinik für Augenheilkunde,
Universitätsklinikum des
Saarlandes UKS
Kirrberger Str. 100,
Gebäude 22,66421 Homburg/
Saar, Deutschland
farah.chr.j@gmail.com

Funding. Open Access funding provided by Projekt DEAL.

\section{Einhaltung ethischer Richtlinien}

Interessenkonflikt. C.J. Farah, B. Seitz, L. Hamon, C. Sourlis und L. Daas geben an, dass kein Interessenkonflikt besteht.

Für diesen Beitrag wurden von den Autoren keine Studien an Menschen oder Tieren durchgeführt. Für die aufgeführten Studien gelten die jeweils dort angegebenen ethischen Richtlinien. Für Bildmaterial oder anderweitige Angaben innerhalb des Manuskripts, über die Patienten zu identifizieren sind, liegt von ihnen und/oder ihren gesetzlichen Vertretern eine schriftliche Einwilligung vor.

Open Access Dieser Artikel wird unter der Creative Commons Namensnennung 4.0 International Lizenz veröffentlicht, welche die Nutzung, Vervielfältigung, Bearbeitung, Verbreitung und Wiedergabe in jeglichem Medium und Format erlaubt, sofern Sie den/die ursprünglichen Autor(en) und die Quelle ordnungsgemäß nennen, einen Link zur Creative Commons Lizenz beifügen und angeben, ob Änderungen vorgenommen wurden.
Die in diesem Artikel enthaltenen Bilder und sonstiges Drittmaterial unterliegen ebenfalls der genannten Creative Commons Lizenz, sofern sich aus der Abbildungslegende nichts anderes ergibt. Sofern das betreffende Material nicht unter der genannten Creative Commons Lizenz steht und die betreffende Handlung nicht nach gesetzlichen Vorschriften erlaubt ist, ist für die oben aufgeführten Weiterverwendungen des Materials die Einwilligung des jeweiligen Rechteinhabers einzuholen.

Weitere Details zur Lizenz entnehmen Sie bitte der Lizenzinformation auf http://creativecommons.org/ licenses/by/4.0/deed.de.

\section{Literatur}

1. Behrens-Baumann W (2009) Diagnostik und Therapie der Keratomykose. Ophthalmologe 106:471-481

2. Behrens-Baumann W, Finis D, MacKenzie C, Roth M, Geerling G (2015) Keratomykose Therapiestandards und aktuelle Entwicklungen. Klin Monatsbl Augenheilkd 232:754-764

3. Borkar DS, Fleiszig SM, Leong Cetal (2013) Association between cytotoxicand invasive pseudomonas aeruginosa and clinical outcomes in bacterial keratitis. JAMA Ophthalmol 131(2):147-153

4. Daas L, Viestenz A, Bischoff M, Hasenfus A, Seitz B (2016) Konfokale Mikroskopie zur Diagnostik der mykotischen Keratitis. Ophthalmologe 113:767-771

5. Daas $L$, Szentmary $N$, Eppig $T$, Langenbucher $A$ Hasenfus A, Roth M, Saeger M, Nölle B, Lippmann B, Böhringer D, Reinhard T, Kelbsch C, Messmer E, Pleyer U, Roters S, Zhivov A, Engelmann K, Schrecker J, Zumhagen L, Thieme H, Darawsha R, Meyer-ter-Vehn T, Dick B, Görsch I, Hermel M, Kohlhaas M, Seitz B (2015) Das Deutsche Akanthamöbenkeratitis-Register. Ophthalmologe 112:752-763

6. Hau SC, Dart JK, Vesaluoma M, Parmar DN, Claerhout I, Bibi K, Larkin DF (2010) Diagnostic accuracy ofmicrobial keratitis with in vivoscanning laser confocal microscopy. Br J Ophthalmol 94(8):982-987

7. lovieno A, Miller D, Ledee DR, Alfonso EC (2014) Cysticidal activity of antifungals against different genotypes of Acanthamoeba. Antimicrob Agents Chemother 58:5626-5628

8. Lin $\mathrm{HC}$, Hsiao $\mathrm{CH}, \mathrm{Ma} \mathrm{DH}$, Yeh LK, Tan HY, Lin MY, Huang SCM (2009) Medical treatment for combined Fusarium and Acanthamoeba keratitis. Acta Ophthalmol 87:199-203

9. Raghavan A, Baidwal S, Vijayaghavan P, Rajeswari S, Rajaraman R, Venkatapathy N, Menon S, Rammohan R (2018) Diagnostic evaluation of cooccurrence of Acanthamoeba and Fungi in Keratitis: A preliminary report. Cornea 37(2):227-234

10. Roth M, Daas L, Renner-Wilde A et al (2019) Das Deutsche Pilz-Keratitis-Register. Ophthalmologe 116:957-966

11. Seitz B, Geerling G, Maier P (2015) Die infektiöse Keratitis: Herpes im Griff, Akanthamöben und Fusarien auf dem Vormarsch. Klin Monatsbl Augenheilkd 232:735-737

\section{Terminankündigung}

2021

DOG 2021

online, 30.09. - 03.10.2021

https://dog-kongress.de/

2022

AAD 2022

Düsseldorf, 15.03. - 19.03.2022

https://aad-kongress.de/

Jahrestagung der BielschowskyGesellschaft 2022

Freiburg i.B., 13.05. - 14.05.2022

http://www.bg2022.de/

DOG 2022

Berlin, 29.09. - 02.10.2022

https://dog-kongress.de/

2023

AAD 2023

Düsseldorf, 21.03. - 25.03.2023

https://aad-kongress.de/

DOG 2023

Berlin, 28.09. - 01.10.2023

https://dog-kongress.de/

\section{4}

AAD 2024

Düsseldorf, 12.03. - 16.03.2024

https://aad-kongress.de/

DOG 2024

Berlin, 10.10. - 13.10.2024

https://dog-kongress.de/ 